\title{
Avaliação da interface entre o componente protético reto e a conexão interna tipo Cone Morse do implante dentário por meio da microscopia eletrônica de varredura
}

\author{
Evaluation of the interface between the straight prosthetic component and the \\ Morse Cone-type internal connection of the dental implant in cross section by \\ scanning electron microscopy
}

\author{
Mariana Bispo COSTA ${ }^{\mathrm{a} *}\left(\mathbb{D}\right.$, Lorena Fernandes FERREIRA ${ }^{\mathrm{a}}(\mathbb{D})$, Wilton Mitsunari TAKESHITA ${ }^{\mathrm{a}}(\mathbb{D}$, \\ Antonio Carlos MARQUETI ${ }^{(1)}$, Cleverson Luciano TRENTOa ${ }^{(1)}$ \\ aUFS - Universidade Federal de Sergipe, Aracaju, SE, Brasil
}

Como citar: Costa MB, Ferreira LF, Takeshita WM, Marqueti AC, Trento CL. Avaliação da interface entre o componente protético reto e a conexão interna tipo Cone Morse do implante dentário por meio da microscopia eletrônica de varredura. Rev Odontol UNESP. 2020;49:e20200033. https://doi.org/10.1590/1807-2577.03320

\begin{abstract}
Resumo
Introdução: A interface implante-pilar protético e a formação dos seus microgaps são aspectos relevantes na transferência das cargas e na resposta biológica, estando ligadas ao sucesso da reabilitação. Objetivo: Avaliar microgaps na interface entre a conexão interna do implante do tipo Cone Morse e a superfície do componente protético por meio da microscopia eletrônica de varredura (MEV). Material e método: Foram utilizados 20 implantes dentários de tamanho 3,75 $\times 11,0 \mathrm{~mm}$ do tipo Cone Morse com seus respectivos pilares protéticos da Singular ${ }^{\circledR}$ (Singular Implants, RN, Brasil). Os munhões retos foram acoplados aos implantes com torque de $32 \mathrm{~N} / \mathrm{cm}^{2}$ e o conjunto resultante foi emergido em base de Resina Epóxi ES260, para permitir secção longitudinal da amostra. As amostras foram analisadas e os microgaps mensurados no MEV (JEOL JCM-5700, MA, USA), e posteriormente os dados foram analisados. Resultado: A média e o desvio padrão dos maiores microgaps foram observados na parte apical do implante nos lados direito e esquerdo, sendo $1,44 \pm 2,68$ e 1,16 $\pm 1,49 \mu \mathrm{m}$, respectivamente. Os menores microgaps foram na parte superior do implante nos lados direito e esquerdo, sendo $0,60 \pm 0,73$ e $0,66 \pm 0,67 \mu \mathrm{m}$, respectivamente. Contudo, no teste de Kruskal-Wallis, não houve diferença estatisticamente significativa entre as regiões dos implantes, tanto para o lado esquerdo $(p=0,692)$ como para o direito $(p=0,865)$. No teste de Mann-Whitney, não houve diferenças estatisticamente significativas entre os lados para as diferentes regiões dos implantes. Conclusão: Mesmo com a presença de microgaps na interface implante-pilar protético, estes apresentam tamanho inferior ao que causaria problemas biológicos e mecânicos. As amostras analisadas quanto à sua compatibilidade de encaixe foram satisfatórias.
\end{abstract}

Descritores: Implantes dentários; pilares protéticos; microscopia eletrônica de varredura.

\begin{abstract}
Introduction: The implant-prosthetic abutment interface and the formation of its microgaps are relevant aspects in load transfer and biological response and are linked to the success of the rehabilitation. Objective: To evaluate microgaps at the internal connection interface of the Cone Morse implant and the prosthetic component surface by means of scanning electron microscopy (SEM). Material and method: Twenty $3.75 \mathrm{x}$ $11.0 \mathrm{~mm}$ Cone Morse dental implants with their respective Singular ${ }^{\circledR}$ prosthetic abutments were used (Singular Implants, RN, Brazil). The straight trunnions were attached to the implants with a $32 \mathrm{~N} / \mathrm{cm} 2$ torque and the ES260 Epoxy Resin-based set emerged to allow longitudinal sectioning of the sample. The samples were analyzed and the microgaps measured in SEM (JEOL JCM-5700, MA, USA), and later the data were analyzed. Result: The mean and standard deviation of the largest microgaps were observed in the apical part of the right and left side $1.44 \pm 2.68$ and $1.16 \pm 1.49 \mu \mathrm{m}$, respectively. The smallest microgaps were on the upper implant right and left sides $0.60 \pm 0.73$ and $0.66 \pm 0.67 \mu \mathrm{m}$, respectively. However, in the Kruskal-Wallis test there was no statistically significant difference between the implant regions, both for the left side $(\mathrm{p}=0.692)$
\end{abstract}


and right side $(\mathrm{p}=0.865)$. The Mann-Whitney test, there were no statistically significant differences between the sides for the different regions of the implants. Conclusion: Even with the presence of microgaps in the implant-post interface, it presents a smaller size than the one presenting biological and mechanical problems. The samples analyzed for their fitting compatibility were satisfactory.

Descriptors: Dental implants; dental abutments; scanning electron microscopy.

\section{INTRODUÇÃO}

Implantes dentários revolucionaram a reabilitação oral em pacientes parcial e totalmente edêntulos ${ }^{1,2}$. São observadas elevadas taxas de sobrevivência e previsibilidade em longo prazo, para implantes endósseos, modalidade de tratamento bem-sucedida na Odontologia1,3-7. No entanto, são observadas falhas ${ }^{3,6-9}$ e a perda óssea ao redor do implante é a mais comum ${ }^{6-8}$. Os fatores que contribuem para perda óssea marginal incluem trauma cirúrgico, perimplantite, sobrecarga oclusal, microinfiltração e anatomia do implante na área da crista óssea ${ }^{3,6,8}$.

A reabilitação pode ser composta por um implante endósseo que se conecta a um pilar transmucoso (duas peças), que recebe a prótese ${ }^{1,4,10,11}$. Independentemente da forma que essa conexão é obtida, é recomendado que seja alcançada a melhor forma de encaixe da interface implante-pilar protético, para favorecer a distribuição de cargas entre os componentes e a resposta biológica, dificultando a proliferação bacteriana ${ }^{1,3,12,13}$. A interface implante-pilar protético tem sido citada como fator significativo na transferência de estresse, nas respostas biológicas adversas ou nas complicações na reconstrução da prótese ${ }^{2,6,10,14}$. Vários fatores relacionados à fabricação dos componentes do implante, bem como o efeito das fases clínica e laboratorial, podem contribuir para um desajuste clínico da prótese $2,4,7,14$. Uma das principais razões para o desajuste de implantes é a presença de microgaps ${ }^{4,8,12}$, que são espaços microscópicos entre o implante e o pilar protético ${ }^{8,10,11,15}$. Em 1990, foi descrito pela primeira vez um vazamento microbiano entre o implante e o pilar protético associado a estes microgaps ${ }^{8}$. Esse termo significa passagem de bactérias e subprodutos tóxicos bacterianos por meio da interface implante-pilar protético, de difícil erradicação, o que leva a uma existência contínua das bactérias, mesmo em pacientes com adequada higiene oral $3,5,6,8,10,15$. Muito embora essa contaminação seja inevitável3,5,15,16, é importante saber que ela pode ocorrer de duas formas: na cirurgia de colocação do pilar protético ou através da exposição da interface ao meio oral para a interface $3,8,9,15$. A problemática encontrada na presença bacteriana na interface implante-pilar protético é a potencial inflamação tecidual perimplantar e, consequentemente, a perda óssea 1,3,4,6,8,9,12,13,15,16, interferindo, assim, na estética e na função da reabilitação ${ }^{6}$.

A presença de micromovimentos entre as partes podem desencadear microgaps que, consequentemente, causam alterações teciduais, desgaste por atrito, afrouxamento do parafuso e fratura dos componentes ${ }^{3-5,8,12,17}$. 0 mercado de implantes busca, cada vez mais, formas de minimizar os microgaps a fim de aumentar a estabilidade entre o implante e o pilar protético, e reduzir a infiltração bacteriana e, consequentemente, a inflamação tecidual8,10,12,16. A confiabilidade e a estabilidade do mecanismo de conexão entre o implante e o pilar protético são um pré-requisito essencial para o sucesso em longo prazo do tratamento ${ }^{10,13}$.

0 valor do torque tem papel importante na formação de microgaps e na infiltração bacteriana $3,5,8,10,11,16$. Um grande valor de torque se traduz no baixo nível de microinfiltração das bactérias, pela redução dos microgaps ${ }^{2,8,11,16}$. E, ainda, os torques de aperto e afrouxamento são os principais fatores na determinação da estabilidade dos pilares, ou seja, a manutenção dos valores de torque do pilar pode impedir o afrouxamento e, consequentemente, a formação de microgaps ${ }^{2,9,13}$.

Estudos têm investigado a capacidade de vedação da interface seja por meio de raios-X (microtomografia), microscopia eletrônica de varredura (MEV) ou microscopia óptica1,4,7,13,17. Estudos in vitro têm demonstrado que o desenho da interface implante-pilar protético pode ter um impacto na quantidade de penetração bacteriana na parte interna do implante ${ }^{13} \mathrm{e}$, ainda, é possível concluir que existe maior contaminação em microgaps maiores ${ }^{12}$. Gois et al.12 relataram que valores de microgaps inferiores a $10 \mu \mathrm{m}$ não produzem efeitos deletérios. 
A conexão do tipo Cone Morse foi desenvolvida com o objetivo de vedar a interface implantepilar protético, evitando, assim, infiltração bacteriana e as consequências das toxinas circulantes na parte interna da conexão, como processo inflamatório e reabsorção óssea ${ }^{10-13,16}$. Além disso, o sistema Cone Morse reduziu notavelmente o centro de rotação, melhorou a estabilidade mecânica e diminuiu a distribuição de tensão no osso marginal ${ }^{8-10}$.

A microscopia eletrônica de varredura (MEV) é a técnica mais recomendada para avaliação e mensuração microscópica de microgaps na interface implante-pilar protético ${ }^{12,18}$. Este recurso fornece alta resolução e grande profundidade de foco do objeto, mantendo-se em posição fixa e predeterminada ${ }^{12}$. Contudo, até o presente momento, poucos trabalhos avaliaram os microgaps em implantes do tipo Cone Morse em MEV. Assim, o objetivo do trabalho foi avaliar e mensurar microgaps na interface criada pela união da superfície da conexão interna do implante do tipo Cone Morse e da superfície do componente protético por meio da MEV.

\section{MATERIAL E MÉTODO}

Este estudo in vitro avaliou a interface entre o implante dentário e o pilar protético, e foi realizado no Departamento de Odontologia de Universidade Federal de Sergipe (UFS), em parceria com o Departamentos de Física e Engenharia de Materiais, em que foi utilizado o microscópio eletrônico de varredura (MEV).

\section{Preparo da Amostra}

Foram utilizados 20 implantes dentários de tamanho 3,75 × 11,0 mm do tipo Cone Morse, com seus respectivos pilares protéticos da Singular® (Parnamirim, RN, Brasil), fabricados em titânio comercialmente puro, que seguem a norma NBR ISO 5832. A preparação das amostras iniciou-se seguindo a orientação do fabricante: os munhões retos foram adaptados, com torque de $32 \mathrm{~N} / \mathrm{cm}^{2}$, ao implante. Após isso, imergiram-se todas as amostras em cubos de Resina Epóxi ES260, a fim de se confeccionar uma base que permitisse a secção longitudinal19,20, que foi realizada na cortadora de precisão Struers Minitom (Struers Inc.Cleveland, USA), com o disco de corte diamantado sob refrigeração (Figura 1).

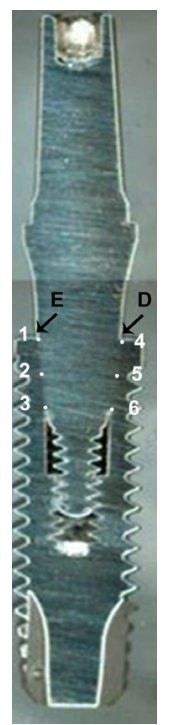

Figura 1. Conjunto implante/pilar protético seccionado para análise no MEV (E-lado esquerdo e D-lado direito da amostra); (1) região superior esquerdo; (2) região média esquerda; (3) região inferior esquerda; (4) região superior direita; (5) região média direita; (6) região inferior direita. 
A secção longitudinal de todas as amostras foi refinada até que cerca de $50 \%$ do volume tivesse sido removido com lixas d'água de diferentes granulações de no 80 a 1.600 (3M, São Paulo, Brasil) e, em seguida, realizou-se polimento com feltro e pasta de alumínio. A medida do corpo de prova seccionado foi criteriosamente verificada em paquímetro digital com sensibilidade de $0,01 \mathrm{~mm}$ (Mitutoyo, São Paulo, Brasil). Estes foram então limpos usando um limpador a vapor (Mondial, São Paulo, Brasil) e seguido por limpeza em banho ultrassônico por 10 minutos (Beijing Ultrasonic Co., Pequim, China). Finalmente, todas as amostras foram lavadas com álcool etílico e secas, com um secador de cabelo (Mondial, São Paulo, Brasil), resultando amostras limpas e secas.

\section{Análise das Amostras}

Para a análise das amostras no microscópio eletrônico de varredura (MEV), a sala encontravase com a temperatura em torno de $20{ }^{\circ} \mathrm{C}$ e umidade do ar inferior a $60 \%$ (avaliação por meio de um termômetro). 0 dispositivo MEV foi ajustado para uma voltagem de aceleração de $15 \mathrm{kV}$. A presença de microgaps entre as conexões internas de implantes e pilares foi assim avaliada. 0 dispositivo MEV empregado foi um JEOL JCM-5700 CARRY SCOPE (Massachusetts, EUA). As imagens do MEV foram obtidas usando o modo de elétrons secundários.

As medições foram feitas por um único pesquisador devidamente calibrado e experiente com o manuseio do MEV, utilizando um ampliador de 3.000 a 10.000 vezes a uma escala de micrômetro $(\mu \mathrm{m})$. Foram registradas seis regiões: inferior, meio e superior, em ambos os lados do implante, e para cada região, foram realizadas três mensurações; portanto, para cada implante, foram obtidas 18 medidas. Como foram analisados 20 implantes, foi obtido um total de 360 medidas, posteriormente tabuladas em Excel 16.0 (Microsoft, Washington, USA).

\section{Análise Estatística}

Todas as informações obtidas foram codificadas e inseridas em um banco de dados. Incialmente foi aplicado o teste de Shapiro-Wilk e, como os dados foram não gaussianos, foram aplicados os testes de Mann-Whitney, para comparação dos lados, e análise de Kruskal-Wallis, para avaliação dos diferentes pontos de registro. 0 nível de significância para todos os testes foi de $5 \%(\mathrm{p} \leq 0,05)$. Os dados foram analisados utilizando a versão do software SPSS 20.0 (IBM Corporation, Armonk, New York, USA).

\section{RESULTADO}

A quantificação dos microgaps, dividida em direita e esquerda, está apresentada na Tabela 1, com seus valores de mínimo, máximo, mediana, desvio padrão e valor de $p$. As análises dos dados aplicando o teste de Mann-Whitney revelaram que não houve diferenças estatisticamente significativas entre os lados esquerdo e direito para as diferentes regiões dos implantes. E, ainda, a análise de Kruskal-Wallis também revelou que não houve diferença estatisticamente significativa entre as regiões dos implantes, tanto para o lado esquerdo $(p=0,692)$ como para o direito $(p=0,865)$.

Tabela 1. Comparação entre os lados direito e esquerdo com valores de mínimo, máximo, mediana, média, desvio padrão e valor de $p$, medidos em micrômetros $(\mu \mathrm{m})$

\begin{tabular}{|c|c|c|c|c|c|c|c|c|c|c|c|}
\hline \multirow{2}{*}{ IIPP } & \multicolumn{5}{|c|}{ Lado esquerdo } & \multicolumn{5}{|c|}{ Lado direito } & \multirow{2}{*}{$\begin{array}{c}\text { Valor de } \\
\mathbf{p}^{1}\end{array}$} \\
\hline & Min & Max & Mediana & Média & DP & Min & $\operatorname{Max}$ & Mediana & Média & DP & \\
\hline Superior & 0,07 & 2,22 & $0,27^{\mathrm{A}}$ & 0,66 & $\pm 0,67$ & 0,06 & 2,67 & $0,27^{\mathrm{A}}$ & 0,60 & $\pm 0,73$ & 0,918 \\
\hline Meio & 0,07 & 2,55 & $0,33^{A}$ & 0,73 & $\pm 0,78$ & 0,11 & 5,42 & $0,21^{\mathrm{A}}$ & 0,89 & $\pm 1,45$ & 0,884 \\
\hline Inferior & 0,07 & 4,41 & $0,27^{\mathrm{A}}$ & 1,16 & $\pm 1,49$ & 0,07 & 5,18 & $0,25^{\mathrm{A}}$ & 1,44 & $\pm 2,68$ & 0,967 \\
\hline Valor de $\mathrm{p}^{2}$ & & & 0,692 & & & & & 0,865 & & & \\
\hline
\end{tabular}

IIPP: interface implante-pilar protético. Min: valor mínimo. Max: valor máximo. DP: desvio padrão. ${ }^{1}$ Teste de Mann-Whitney U. ${ }^{2}$ Teste de Kruskal-Wallis. p<0,05. Letras diferentes denotam resultados significativamente diferentes. Letras maiúsculas comparam valores na vertical. 


\section{DISCUSSÃO}

Implantes dentários são excelentes opções para a reabilitação oral ${ }^{1,2}$ devido à sua alta taxa de sucesso em longo prazo ${ }^{1,3-7}$, embora existam falhas ${ }^{3,6-9}$. A conexão entre o implante dentário e o pilar protético deve ser o mais hermético possível, afim de evitar a presença de robustos microgaps, que geram interferências biológicas e mecânicas que culminam na perda óssea perimplantar1,3,4,6,8,9,12,13,15,16. As imagens da MEV obtidas no nosso trabalho de pesquisa revelaram a presença de microgaps em ambos os lados dos implantes, com maiores valores na região inferior.

Este resultado corrobora em parte com o trabalho de Duraisamy et al.19, pois também observaram os maiores valores de microgaps na região inferior e os menores, na região superior. No entanto, os valores médios dos microgaps foram bem maiores do que o presente trabalho de pesquisa e isto pode ser explicado pelo fato de os implantes serem de marcas e design diferentes. No trabalho de Lopes et al. $^{20}$, que utilizaram implantes do tipo Cone Morse da Neodent ${ }^{\circledR}$, apresentaram-se valores médios maiores do que os verificados em nossa pesquisa. Contudo, os valores máximo e mínimo foram semelhantes. Além disso, houve discordância de Ranieri et al. ${ }^{16}$, que apresentaram valores de microgaps maiores, do que os observados em nossa pesquisa. Entretanto, as marcas estudadas foram Ankylos®, Neodent $₫$, Conexão $®$ e Osteofit $₫$.

Com isso, no estudo de Ceruso et al.21, realizou-se uma revisão sistemática em que analisaram as características de microgaps, infiltração bacteriana e perda óssea de três tipos diferentes de pilar de implantes. Com relação aos microgaps, os implantes do tipo Cone Morse apresentaram valores de microgaps menores do que $10 \mu \mathrm{m}$, corroborando com os dados desta pesquisa. Contudo, outros estudos são necessários para elucidar o real efeito in vivo dos microgaps.

É sabido que os microgaps estão relacionados à infiltração bacteriana e uma grande quantidade de bactérias pode colonizar essa região, dentre elas, Passos et al. ${ }^{3}$ citam Streptoccocus sanguis (tamanho médio varia em torno de 0,8 $\mu \mathrm{m}$ ) e corroboram com Koutouzis et al.13, em que ambos os estudos citam a Escherichia coli (tamanho médio variando entre 1,1 $\mu \mathrm{m}$ e 1,5 $\mu \mathrm{m}$ de diâmetro, e $2 \mu \mathrm{m}$ e $6 \mu \mathrm{m}$ de comprimento). Contudo, não podemos considerar somente o tamanho bacteriano como fator para determinar a capacidade de colonizar essa região.

Com isso, no presente trabalho de pesquisa, apenas na região superior da interface entre o implante dentário e o pilar protético verificada, não é possível a proliferação bacteriana dessas espécies. Portanto, conhecer o tamanho da interface pode permitir compreender o potencial da colonização bacteriana, porém não fornece informações acerca da possibilidade de passagem de fluido através da interface implante-pilar protético e, para corroborar com isto, no trabalho de Lorenzoni et al. ${ }^{1}$, foi possível observar a passagem de fluidos pelos microgaps. Ou seja, corroborando com o que Passos et al. ${ }^{3}$ relataram, mesmo em interfaces muito bem ajustadas, não é possível impedir o vazamento e a colonização bacteriana nesta região.

A fim de complementar, Alves et al. ${ }^{10}$ afirmaram que, se as bactérias não conseguirem superar a conexão entre o pilar protético e o implante de dentro para fora, esses microrganismos também serão incapazes de transpor essa área na direção oposta. Portanto, a região mais importante para impedir a proliferação bacteriana é a região superior da interface entre o implante dentário e o pilar protético. Aloise et al. ${ }^{15}$ relataram que, independentemente do sistema de ativação do Cone Morse, é possível observar vazamento bacteriano ao longo da interface implante-pilar protético, embora este fosse menor que nos demais sistemas de conexão implante-prótese.

No trabalho de Lorenzoni et al. ${ }^{1}$, recomendaram cautela quando somente uma técnica é utilizada para avaliar a interface, uma vez que se acredita que existam variações nos tamanhos dos microgaps no raio do implante, o que pode ser notado neste trabalho também, no qual verificamos que, na porção superior da conexão, os microgaps eram menores que na porção inferior do conjunto. Com relação às causas dos microgaps, no trabalho de Coelho et al. ${ }^{4}$, foi relatado que sua ocorrência fora devida à usinagem imprecisa do implante. 
Coelho et al. ${ }^{4}$ relataram que a presença de um microgap crescente em função do raio provavelmente afeta a estabilidade da prótese em longo prazo de maneira diferente de uma distância uniforme entre o implante e o pilar protético. Em nosso trabalho, foi possível observar o aumento do microgap em função da redução do raio do implante, ou seja, afetando menos a estabilidade da prótese.

Um achado importante que pode se verificar em todas amostras é que os microgaps não foram regulares em toda extensão do conjunto, o que sugere que esses microgaps não se comunicam entre si e sequer se comunicam com o meio externo, bem como verificou-se também um tipo de ponte de oxidação entre implante e componente protético, que quando formado nos direciona para um tipo de vedação físico-química semelhante a uma soldadura a frio entre as partes (Figura 2).

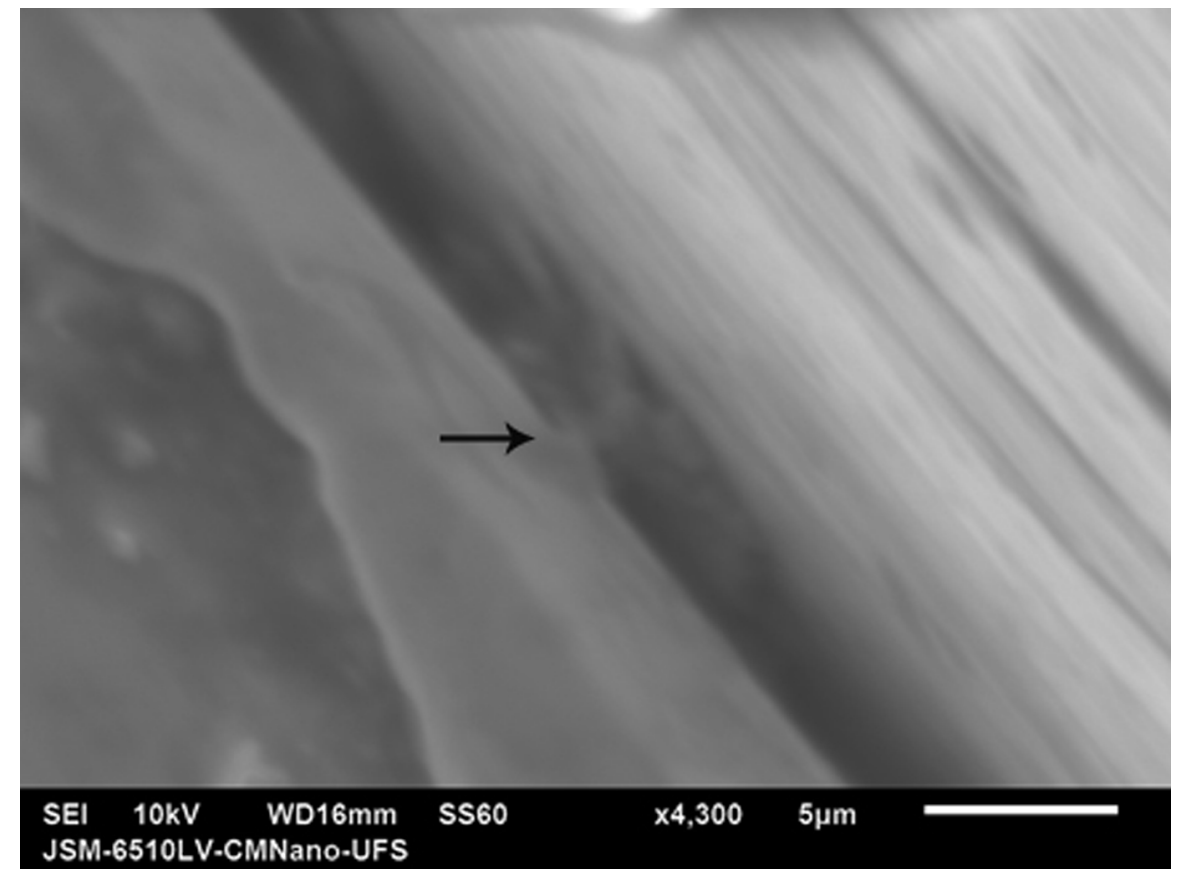

Figura 2. Seta indicando a ponte de oxidação entre implante e o pilar protético.

Com relação ao tamanho do microgap, Gois et al.12, Duraisamy et al. ${ }^{19}$ Zanardi et al. ${ }^{22}$ relataram que microgaps menores que $10 \mu \mathrm{m}$ não produzem efeitos deletérios. Ao comparar esta conclusão ao nosso estudo, é possível concluir que os valores de microgaps foram satisfatórios, uma vez que se apresentam inferiores a $10 \mu \mathrm{m}$.

Por fim, outros estudos devem ser realizados comparando outras marcas de implantes, com outras técnicas de avaliação da interface do implante e estudos in vivo, verificando o comportamento da interface implante-pilar protético. Seria interessante focar, principalmente, no que concerne a um dos achados do presente trabalho de pesquisa, uma ponte de oxidação entre o implante e o pilar protético, que poderia sugerir um melhor vedamento. Assim, outros estudos in vivo e in vitro são necessários, para melhor elucidar esse achado.

\section{CONCLUSÃO}

Os maiores microgaps foram encontrados na região inferior da interface implante-pilar protético e, em contraposição, os menores microgaps foram encontrados na região superior. Embora exista a presença de microgaps na interface implante-pilar protético, estes apresentam tamanho inferior ao tamanho que poderia causar problemas biológicos e mecânicos. 


\section{REFERÊNCIAS}

1. Lorenzoni FC, Coelho PG, Bonfante G, Carvalho RM, Silva NRFA, Suzuki M, et al. Sealing capability and SEM observation of the implant-abutment interface. Int J Dent. 2011;2011:864183. http://dx.doi.org/10.1155/2011/864183. PMid:21754934.

2. Gehrke SA, Shibli JA, Aramburú JS Jr, Sánchez de Val JEM, Calvo-Girardo JL, Dedavid BA. Effects of different torque levels on the implant-abutment interface in a conical internal connection. Braz Oral Res. 2016;30(1):e40. http://dx.doi.org/10.1590/1807-3107BOR-2016.vol30.0040. PMid:26981760.

3. Passos SP, Gressler May L, Faria R, Özcan M, Bottino MA. Implant-abutment gap versus microbial colonization: clinical significance based on a literature review. J Biomed Mater Res B Appl Biomater. 2013 Oct;101(7):1321-8. http://dx.doi.org/10.1002/jbm.b.32945. PMid:23661560.

4. Coelho AL, Suzuki M, Dibart S, Da Silva N, Coelho PG. Cross-sectional analysis of the implant abutment interface. J Oral Rehabil. 2007 Jul;34(7):508-16. http://dx.doi.org/10.1111/j.13652842.2007.01714.x. PMid:17559619.

5. Scarano A, Valbonetti L, Degidi M, Pecci R, Piattelli A, de Oliveira PS, et al. Implant-abutment contact surfaces and microgap measurements of different implant connections under 3-dimensional X-Ray microtomography. Implant Dent. 2016 Oct;25(5):656-62. http://dx.doi.org/10.1097/ID.0000000000000465. PMid:27551879.

6. Dias ECLCM, Sperandio M, Napimoga MH. Association between implant-abutment microgap and implant circularity to bacterial leakage: an in vitro study using tapered connection implants. Int J Oral Maxillofac Implants. 2018 May-June;33(3):505-11. http://dx.doi.org/10.11607/jomi.5836. PMid:28938028.

7. Jörn D, Kohorst P, Besdo S, Borchers L, Stiesch M. Three-dimensional nonlinear finite element analysis and microcomputed tomography evaluation of microgap formation in a dental implant under oblique loading. Int J Oral Maxillofac Implants. 2016 May-Jun;31(3):e32-42. http://dx.doi.org/10.11607/jomi.4179. PMid:27183080.

8. Liu Y, Wang J. Influences of microgap and micromotion of implant: abutment interface on marginal bone loss around implant neck. Arch Oral Biol. 2017 Nov;83:153-60. http://dx.doi.org/10.1016/j.archoralbio.2017.07.022. PMid:28780384.

9. Romanos GE, Biltucci MT, Kokaras A, Paster BJ. Bacterial composition at the implant-abutment connection under loading in vivo. Clin Implant Dent Relat Res. 2016 Feb;18(1):138-45. http://dx.doi.org/10.1111/cid.12270. PMid:25195711.

10. Alves DCC, Carvalho PSP, Martinez EF. In vitro microbiological analysis of bacterial seal at the implantabutment interface using two morse taper implant models. Braz Dent J. 2014 Feb;25(1):48-53. http://dx.doi.org/10.1590/0103-6440201302178. PMid:24789292.

11. Alqutaibi AY, Aboalrejal AN. Microgap and micromotion at the implant abutment interface cause marginal bone loss around dental implant but more evidence is needed. J Evid Based Dent Pract. 2018;18(2):171-2. http://dx.doi.org/10.1016/j.jebdp.2018.03.009. PMid:29747801.

12. Gois DM Fo, Gois-Santos VT, Silva RS, Marqueti AC, Cortes ARG, Trento CL. Evaluation of sealing between abutment and inner connection of cone morse dental implant: microgaps between implant and abutment. Clin Lab Res Dent. 2018;2018:1-6. https://doi.org/10.11606/issn.23578041.clrd.2018.147797.

13. Koutouzis T, Gadalla H, Lundgren T. Bacterial colonization of the implant-abutment interface (IAI) of dental implants with a sloped marginal design: an in-vitro study. Clin Implant Dent Relat Res. 2016 Feb;18(1):161-7. http://dx.doi.org/10.1111/cid.12287. PMid:25623033.

14. Barbosa GAS, Neves FD, Mattos MGC, Rodrigues RCS, Ribeiro RF. Implant/abutment vertical misfit of one-piece cast frameworks made with different materials. Braz Dent J. 2010;21(6):515-9. http://dx.doi.org/10.1590/S0103-64402010000600006. PMid:21271041. 
15. Aloise JP, Curcio R, Laporta MZ, Rossi L, Silva AMA, Rapoport A. Microbial leakage through the implant - abutment interface of morse taper implants in vitro. Clin Oral Implants Res. 2010 Mar;21(3):328-35. http://dx.doi.org/10.1111/j.1600-0501.2009.01837.x. PMid:20074246.

16. Ranieri R, Ferreira A, Souza E, Arcoverde J, Dametto F, Gade-Neto C, et al. The bacterial sealing capacity of morse taper implant-abutment systems in vitro. J Periodontol. 2015;86(5):696-702. http://dx.doi.org/10.1902/jop.2015.140623. PMid:25658884.

17. Grobecker-Karl T, Karl M. correlation between micromotion and gap formation at the implantabutment interface. Int J Prosthodont. 2017 Mar/Apr;30(2):150-2. http://dx.doi.org/10.11607/ijp.5086. PMid:28267824.

18. Souza RS, Suffredini IB, Cortizo DL, Larsson A, Nannmark U, Dib LL. In vitro analysis of the implantabutment interface connection and bacterial infiltration in two extraoral implant models. Int J Oral Maxillofac Implants. 2020 Jan/Feb;35(1):63-9. http://dx.doi.org/10.11607/jomi.7692. PMid:31923290.

19. Duraisamy R, Krishnan CS, Ramasubramanian H, Sampathkumar J, Mariappan S, Navarasampatti Sivaprakasam A. Compatibility of nonoriginal abutments with implants: evaluation of microgap at the implant-abutment interface, with original and nonoriginal abutments. Implant Dent. 2019 Jun;28(3):289-95. http://dx.doi.org/10.1097/ID.0000000000000885. PMid:31124826.

20. Lopes PA, Carreiro AFP, Nascimento RM, Vahey BR, Henriques B, Souza JCM. Physicochemical and microscopic characterization of implant-abutment joints. Eur J Dent. 2018 Jan-Mar;12(1):100-4. http://dx.doi.org/10.4103/ejd.ejd_3_17. PMid:29657532.

21. Ceruso FM, Barnaba P, Mazzoleni S, Ottria L, Gargari M, Zuccon A, et al. Implant-abutment connections on single crowns: a systematic review. Oral Implantol. 2017 Oct-Dec;10(4):349-53. http://dx.doi.org/10.11138/orl/2017.10.4.349. PMid:29682251.

22. Zanardi PR, Costa B, Stegun RC, Sesma N, Mori M, Laganá DC. Connecting accuracy of interchanged prosthetic abutments to different dental implants using scanning electron microscopy. Braz Dent J. 2012 Oct;23(5):502-7. http://dx.doi.org/10.1590/S0103-64402012000500006. PMid:23306225.

\section{CONFLITOS DE INTERESSE}

Os autores declaram não haver conflitos de interesse.

\section{*AUTOR PARA CORRESPONDÊNCIA}

Mariana Bispo Costa, UFS - Universidade Federal de Sergipe, Rua Cláudio Batista, s/n, Palestina, 49060-108 Aracaju SE, Brasil, e-mail: marianabispocosta@gmail.com

Recebido: Maio 5, 2020

Aprovado: Agosto 13, 2020 